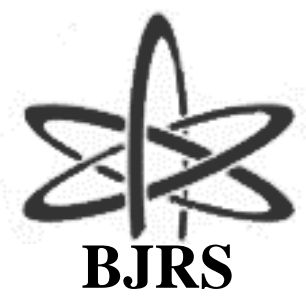
BRAZILIAN JOURNAL
$\mathrm{OF}$
RADIATION SCIENCES
09-01A (2021) 01-18

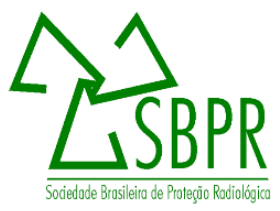

\title{
Spatial resolution of a preclinical PET tomograph
}

\author{
${ }^{1,2}$ Souza, G.A.C., ${ }^{1,2}$ Gontijo, R.M.G., ${ }^{1}$ Silva, J.B., ${ }^{1,2}$ Mamede, M., ${ }^{1}$ Ferreira, A.V. \\ ${ }^{1}$ Centro de Desenvolvimento da Tecnologia Nuclear (CDTN/CNEN - MG) \\ Av. Presidente Antônio Carlos, 6.627 \\ 31270-901 Belo Horizonte, MG \\ guilherme.cavalcante@cdtn.br \\ ${ }^{2}$ Departamento de Anatomia e Imagem - (IMA/FM - UFMG) \\ Universidade Federal de Minas Gerais \\ 30130-100 Belo Horizonte, MG
}

\begin{abstract}
Positron emission tomography (PET) is an important molecular image modality and its application in preclinical research has increased during the last decades. Thus, in laboratory practice, it is important to implement a quality control of the equipment, since intrinsic factors influence the image quality. The objective of this work was to perform and implement spatial resolution tests for the small animal PET scanner of the Molecular Imaging Laboratory, LIM/CDTN. Empirically, the spatial resolution of a PET scanner can be determined from the measurement of point or linear sources and FWHM (full width half maximum) analysis of the PET image. In this work, a point source of ${ }^{22} \mathrm{Na}$ and a hot rod style phantom filled with the ${ }^{18} \mathrm{~F}$-FDG solution were used. Acquisition and reconstruction of images were performed with the LabPET 1.12.1 software, provided by the equipment manufacturer. PeakFit ${ }^{\circledR}$ and Amide software were used to perform images post-processing. The results indicate that the PET scanner spatial resolution value is compatible with values reported in an international study performed on similar equipment.
\end{abstract}

Keywords: Spatial resolution, small animal PET scanner, image quality control. 


\section{INTRODUCTION}

Positron emission tomography (PET) is one of the most important diagnostic and therapeutic methods in Nuclear Medicine (MN) due to its sensitivity in the detection of functional alterations at the molecular level. The Molecular Imaging Laboratory (LIM) of the Nuclear Technology Development Center (CDTN/CNEN) possesses a small animal PET scanner (LabPET SOLO 4, Triumph ${ }^{\mathrm{TM}}$ ) which is used for preclinical studies concerning new radiopharmaceuticals development or new applications of traditional radiopharmaceuticals.

Several intrinsic parameters of PET scanners, such as noise, scattered radiation, contrast, sensitivity and spatial resolution, interfere with the quality of the acquired PET image [1]. In general, commercial small animal PET scanners have an image resolution between 1.3 and $2 \mathrm{~mm}$ [2]. The spatial resolution of a PET scanner is a measure of the equipment's ability to faithfully reproduce the image of an object, clearly showing variations in the distribution of radioactivity. It is empirically defined as the minimum distance between two points in a PET image that can be detected [1].

The spatial resolution of PET scanner is affected by many factors: (i) detector size, (ii) positron range, (iii) non-colinearity, (iv) image reconstruction method, (v) detector location [1]. Intrinsic resolution $\left(\mathrm{R}_{\mathrm{i}}\right)$ of scintillation detector greatly affects the PET scanner spatial resolution. In general, for multi-detectors PET scanners, $R_{i}$ is given by $d / 2$, where $d$ is the detector size. The positron range $\left(R_{p}\right)$ generates an error in the localization of the true position of the positron emission since coincidence detection is related to the location of positron annihilation. Non-colinearity $\left(R_{a}\right)$ degrades the spatial resolution and occurs when the two $511 \mathrm{keV}$ photons deviate from the exact $180^{\circ}$ position due to some small residual momentum of the positron at the end of the positron range. The error due to the image reconstruction method $\left(\mathrm{K}_{\mathrm{r}}\right)$ introduces an additional degradation of the spatial resolution. $\mathrm{K}_{\mathrm{r}}$ is usually a factor of 1.2-1.5 depending on the chosen method. The use of block detectors causes an error $\left(\mathrm{R}_{1}\right)$ at the location of the detector by $\mathrm{X}, \mathrm{Y}$ analysis - it may amount to $2.2 \mathrm{~mm}$ for BGO detectors. Therefore, combining these factors, the overall spatial resolution $\left(\mathrm{R}_{\mathrm{t}}\right)$ of a PET scanner is given by [1]: 


$$
R_{t}=K_{r} \sqrt{R_{i}^{2}+R_{p}^{2}+R_{a}^{2}+R_{l}^{2}}
$$

More information concerning PET spatial resolution, its fundamental limits and dependence on detectors type may be found in the literature [3-6].

Empirically, the spatial resolution of PET scanners can be determined from measurement of point or linear sources and analysis of the FWHMs (Full Width Half Maximum) from respective images. In this sense, a standard characterizing the spatial resolution of small animal PET scanners was published by the National Electrical Manufacturers Association (NEMA) [7]. The publication recommends the characterization of the spatial resolution by means of the determination of the parameters (i) FWHM and (ii) FWTM (full width at tenth maximum) of the reconstructed image of $a^{22} \mathrm{Na}$ point source along radial, tangential and axial directions. NEMA NU 4-2008 publication establishes that image reconstruction must be done with no smoothing using the filtered backprojection (FBP) reconstruction algorithm. Due to the linearity of the FBP reconstruction algorithm, the measured spatial resolution is independent of activity distribution in the background [4]. It is important to note that NEMA NU 4-2008 publication points that the measurement does not represent a real condition of animal imaging, but allows a reproducible comparison among scanners, indicating the highest achievable performance for each operation mode [7].

The objective of this work was to characterize the spatial resolution of the LIM/CDTN small animal PET scanner. Although literature presents studies about the characterization of parameters of Triumph ${ }^{\mathrm{TM}}$ PET systems [8-11], there is a lack of studies on the first generation of these systems. Spatial characterization performed in this work followed as closely as feasible the standard method recommended by NEMA NU 4-2008 publication. An additional method was also performed.

Furthermore, the spatial resolution tests performed in this work were incorporated into the Quality Assurance Program (QAP) for Molecular Images of the LIM/CDTN. Recent work revealed that there are few small animal PET scanners installed in Brazil and the most do not have a quality assurance program implemented. Despite the absence of QAPs, there is a unanimous agreement between small animal PET researchers in Brazil on their importance in the preclinical molecular imaging research field [12]. 


\section{MATERIALS AND METHODS}

In the development of this work, the LIM/CDTN small animal PET scanner, the commercial QMR Micro-PET Hot-Rod Phantom (Figure 1) and a ${ }^{22} \mathrm{Na}$ point source (Figure 2) were used in PET imaging.

\subsection{Materials}

\subsubsection{Small animals PET scanner}

The PET scanner of the LIM/CDTN belongs to the Triumph ${ }^{\mathrm{TM}}$ LabPET SOLO 4 platform, manufactured by Gamma Medica / GE Healthcare. The PET scanner is routinely used in preclinical studies to acquire metabolic and functional images of small animal organs and tissues.

LabPET SOLO 4 system consists of a stationary gantry with 1536 detection channels. The detectors are composed of two different types of scintillators, LYSO, Lithium OrthoSilicate with Lithium $\left(\mathrm{Lu}_{1.9} \mathrm{Y}_{0.1} \mathrm{SiO}_{5}\right)$, and LGSO, Lithium OrthoSilicate with Gadolinium $\left(\mathrm{Lu}_{0.4} \mathrm{Gd}_{1.6} \mathrm{SiO}_{5}\right)$, optically coupled to avalanche photodiode detectors (APD). The detector array is arranged in continuous rings with a $15.6 \mathrm{~cm}$ diameter and an axial field of view (FOV) of $3.7 \mathrm{~cm} \mathrm{[13].} \mathrm{PET}$ images are acquired using an energy window in the range of $250-650 \mathrm{keV}$ and coincidence events with a time window of $22 \mathrm{~ns}$. The system can operate in dynamic (temporal) or static (spatial) mode.

\subsubsection{Hot Rod Phantom}

QMR Micro-PET Hot Rod Phantom (Figure 1) is a PMMA device, specially designed to evaluate the spatial resolution of small animal PET scanners [14]. The phantom has a height of 7 $\mathrm{cm}$, a diameter of $3.5 \mathrm{~cm}$ and a volume of approximately $26 \mathrm{~cm}^{3}$. Its interior contains three discs, one of them with fillable channels. These channels are arranged into six groups (G1 to G6) of different diameters: $2 \mathrm{~mm}(\mathrm{G} 1), 1.5 \mathrm{~mm}(\mathrm{G} 2), 1.2 \mathrm{~mm}(\mathrm{G} 3), 1 \mathrm{~mm}(\mathrm{G} 4), 0.8 \mathrm{~mm}$ (G5) and $0.6 \mathrm{~mm}$ (G6). In each group, the spacing between the channels corresponds to twice the diameter of the channels. 
Figure 1: Left: QMR Micro-PET Hot Rod Phantom; Center: Details of Phantom components; Right: Disc with fillable channels arranged into six groups of different diameters [14].

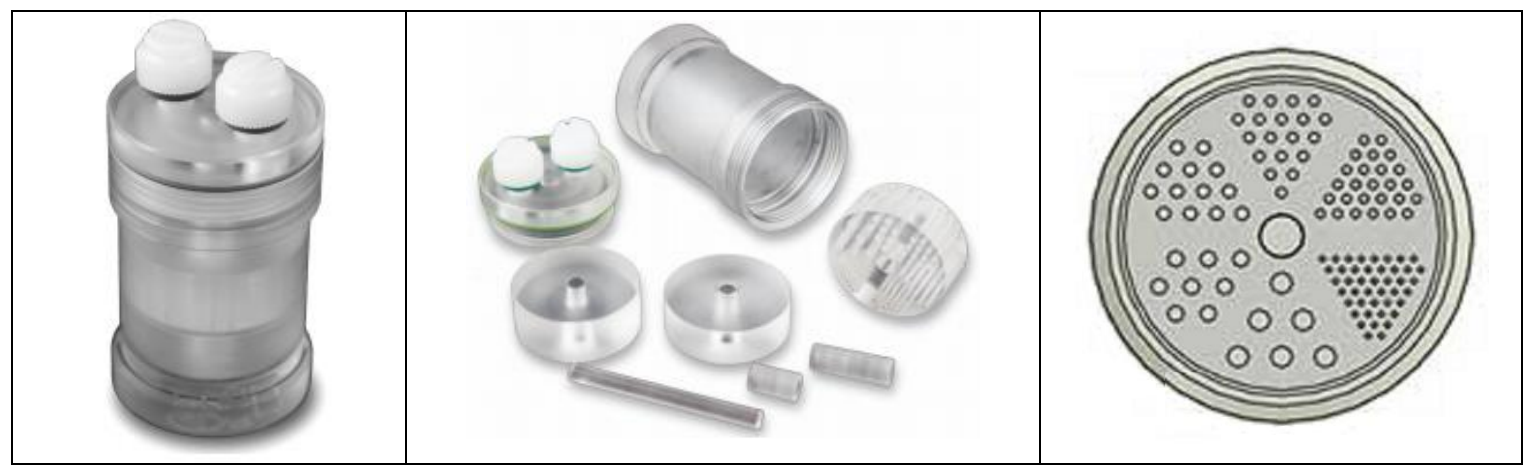

\subsection{3 ${ }^{22}$ Na point source}

The ${ }^{22} \mathrm{Na}$ point source (Figure 2) is embedded in a PMMA acrylic cube and has an active diameter of $0.25 \mathrm{~mm}$. Its activity is $1.154 \mathrm{MBq}$ on $2018 / 12 / 01$, according to the Certificate of Calibration provided by the manufacturer. ${ }^{22} \mathrm{Na}$ is a positron emitter with a half-life of 2.6 years.

Figure 2: ${ }^{22}$ Na point source *

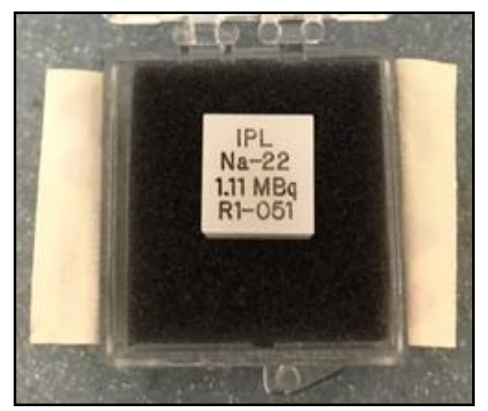

*Authors photo gallery.

\subsection{Image Acquisition Procedures}

\subsubsection{Hot Rod Phantom Images}

For images acquisition, the Hot Rod phantom was filled with ${ }^{18}$ F-FDG (Radioaglic $\left.®\right)$ supplied by the CDTN/CNEN Radiopharmaceuticals Research and Production Unit (UPPR/CDTN). The phantom was disposed of in such a way that the region of interest (fillable channels, Figure 1) was positioned in the centre of the FOV. PET images were acquired and reconstructed using LabPET 
software 1.12.1 (provided by the equipment manufacturer). Table 1 summarizes experimental conditions for the acquisition and reconstruction of the PET images.

Table 1: Experimental conditions for acquisition and reconstruction of the PET images of the Hot Rod Phantom.

\begin{tabular}{c|c|c}
\hline \multirow{2}{*}{ Acquisition } & Acquisition time & Value \\
\hline \multirow{7}{*}{ Reconstruction } & Activity & 1 hour \\
& Acquisition mode & $60 \mathrm{MBq}$ \\
& Number of bed positions & Spatial (static) \\
\hline \multirow{4}{*}{ Transversal FOV } & $46 \mathrm{~mm}$ \\
& Mumber of iterations & MLEM-3D \\
& High-resolution mode & 20 \\
& Num & No \\
\hline
\end{tabular}

Reconstructed PET images were analyzed using the PMOD® software. In this step, graphs containing the line profile of PET signal intensity across the fillable channels were generated for each phantom group. Afterwards, obtained line profiles were analyzed using the PeakFit ${ }^{\circledR}$ software to determine FWHMs of the peaks of interest.

\subsection{2 ${ }^{22}$ Na Point Source Images}

Acquisitions and reconstructions of ${ }^{22} \mathrm{Na}$ point source PET images were carried out as determined by the NEMA NU 4-2008 publication [7]. A source positioner was developed and later fixed to the mice bed (Figure 3 - left). Posteriorly, the positioner and point source were aligned with the centre of the FOV (Figure 3 - right). PET images were acquired in two stages. First, acquisitions were done with the source located at the axial centre of the FOV and then acquisitions were done with the source located at a one-fourth of the axial FOV from the centre of the axial FOV. For these two stages, images were acquired at radial distances from the centre: $0 \mathrm{~mm}, 5 \mathrm{~mm}, 10 \mathrm{~mm}, 15 \mathrm{~mm}$ and $25 \mathrm{~mm}$. PET images acquisition time was defined in such a way that the number of prompt 
counts was greater than $10^{5}$ counts for each image, as recommend by NEMA NU 4-2008 publication [7].

Figure 3: Left: ${ }^{22} \mathrm{Na}$ point source positioner schema;

Right: Positioner and ${ }^{22} \mathrm{Na}$ point source positioned at the axial centre of the FOV.
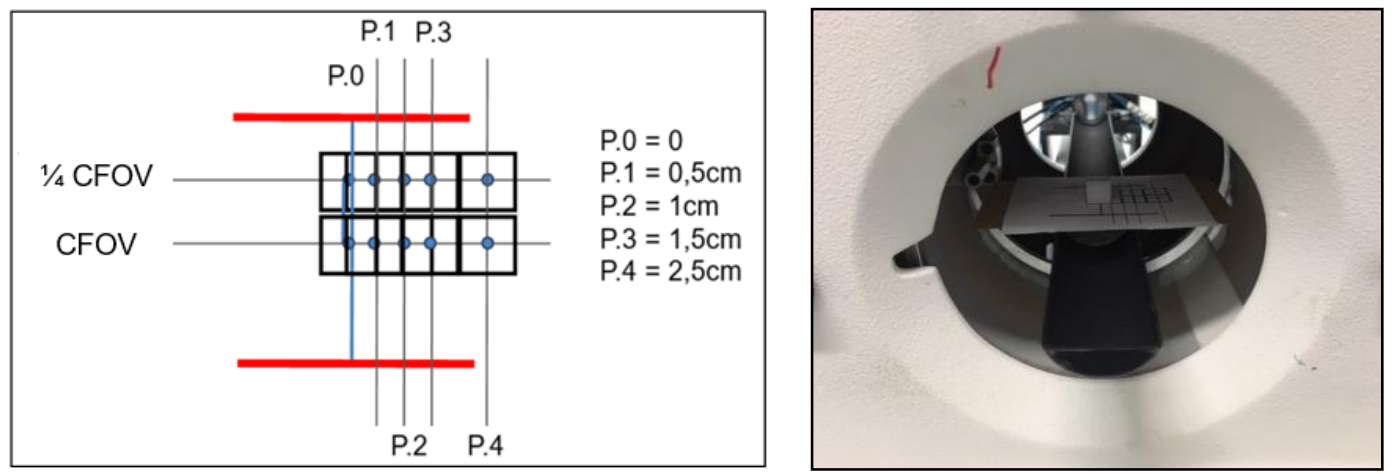

PET images were acquired and reconstructed using LabPET software 1.12.1, provided by the equipment manufacturer. Table 2 summarizes the experimental conditions for the acquisition and reconstruction of the PET images.

Table 2: Experimental conditions for acquisition and reconstruction of the PET images of the ${ }^{22}$ Na point source.

\begin{tabular}{c|c|c}
\hline \multirow{4}{*}{ Acquisition } & Acquisition time & Value \\
\hline \multirow{5}{*}{ Reconstruction } & Activity & $2 \mathrm{~min}$ \\
& Number of acquisitions & $1,1 \mathrm{MBq}$ \\
& Transversal FOV & 10 \\
\cline { 2 - 2 } & Mumber of iterations & $60 \mathrm{~mm}$ \\
& Num & MLEM-3D and FBP-2D \\
& High-resolution mode & No \\
\hline
\end{tabular}


Although NEMA NU 4-2008 publication recommends using analytical FBP reconstruction algorithm to spatial resolution characterization [7], additional analyses using iterative MLEM-3D method were also performed (Table 2). The irregular crystal spacing of the LabPET scanner in both the azimuthal and axial directions results in sub-optimal reconstruction with the FBP algorithm, which requires evenly distributed projection data $[10,11]$. The data resampling and interpolation for rebinning measured data onto projections with regular spacing introduce inaccuracies and artefacts that degrade the resolution and make it uneven across the FOV $[10,11]$. In this sense, iterative reconstruction methods are more adapted to resolve this issue [10, 11]. However, iterative reconstruction algorithms are nonlinear and the non-negativity constraint can artificially enhance the apparent spatial resolution if a point source image is reconstructed without any background [4]. As MLEM-3D is the iterative method used routinely for image reconstruction at LIM/CDTN, this method was chosen for the additional spatial resolution characterization performed in this work. These additional analyses aimed to evaluate the influence of the image reconstruction method in the spatial resolution measured using a point source.

Reconstructed PET images were analyzed using the Amide software. Since the LabPET system does not permit the extraction and handling of sinograms, image analyses were adapted to follow as closely as feasible the NEMA NU 4-2008 publication. In this step, for each image, three graphs were generated - one for each direction (radial, tangential and axial). From these graphs, the line profile of the PET signal intensity along the ${ }^{22} \mathrm{Na}$ point source was obtained. Afterwards, obtained line profiles were analyzed using a Gaussian fit to determine FWHMs and FWTMs of the ${ }^{22} \mathrm{Na}$ point source PET image.

Table 3 summarizes the geometry conditions of the line profiles of ${ }^{22} \mathrm{Na}$ point source placed at different positions in the scanner FOV.

Table 3: Geometry parameters of line profiles of ${ }^{22} \mathrm{Na}$ point source PET images.

\begin{tabular}{cccc}
\hline Line profile & Direction & Plan & Angle \\
\hline L1 & Radial & Transversal & $0^{\circ}$ \\
L2 & Tangential & Transversal & $90^{\circ}$ \\
L3 & Axial & Sagittal or Coronal & $90^{\circ}$ \\
\hline
\end{tabular}




\section{RESULTS AND DISCUSSION}

\subsection{Hot Rod Phantom Images}

Figure 5 shows a PET image of the Hot Rod phantom in an axial view. A qualitative analysis of this image reveals that the system can resolve the PET signals of each fillable channel from groups G1, G2 and G3 while for G4 to G6 group the system does not have sufficient spatial resolution to distinguish the fillable channels.

Figure 5: PET image of the QMR Micro-PET Hot-Rod Phantom (axial view).

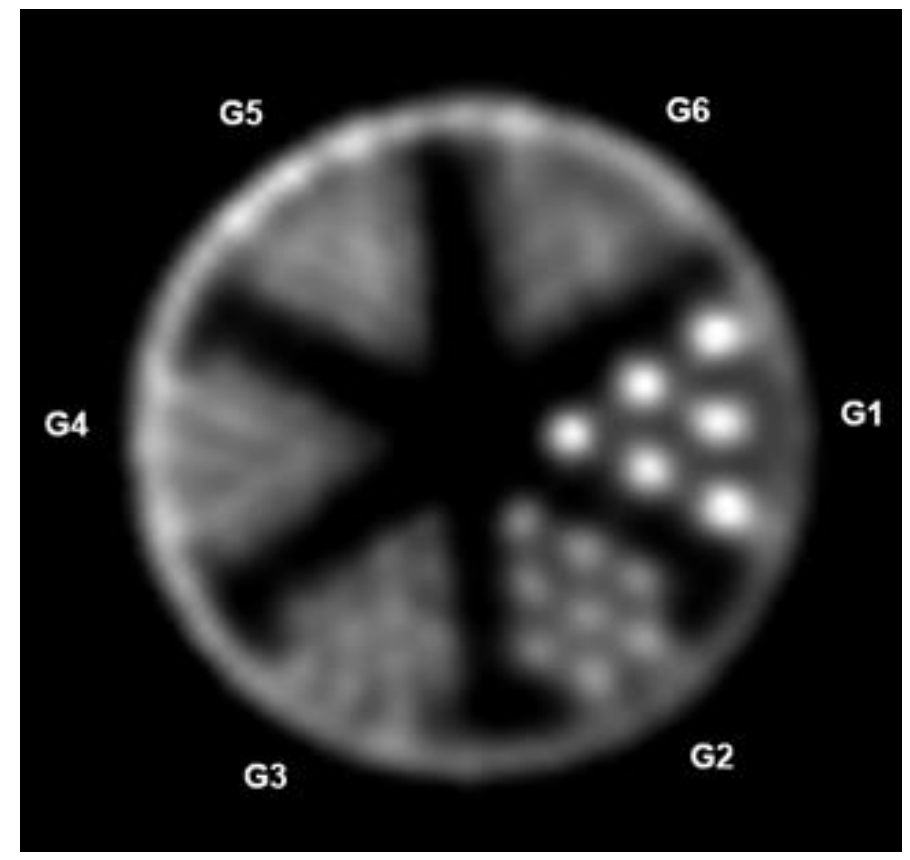

Figure 6 illustrates the obtaining and analysis of the line profile for Group G1. First, a line passing through the centre of the fillable channels was defined (Figure 6A) and then the line profile of the PET signal was obtained (Figure 6B). These steps were performed using PMOD® software. Afterwards, peaks presents in the line profile were deconvoluted (Figure 6C) using PeakFit® software. In the Figure, the left peak refers to the edge signal of the Hot Rod phantom, while the peaks P2, P3 and P4 refer to the fillable channels of $2.0 \mathrm{~mm}$ from Group G1. 
Figure 6: PET image analysis. A: Definition of the line passing through the centre of the G1 fillable channels (P2, P3, P4). B: Line profile of G1 PET image. C: Peaks deconvolution from G1 line profile.

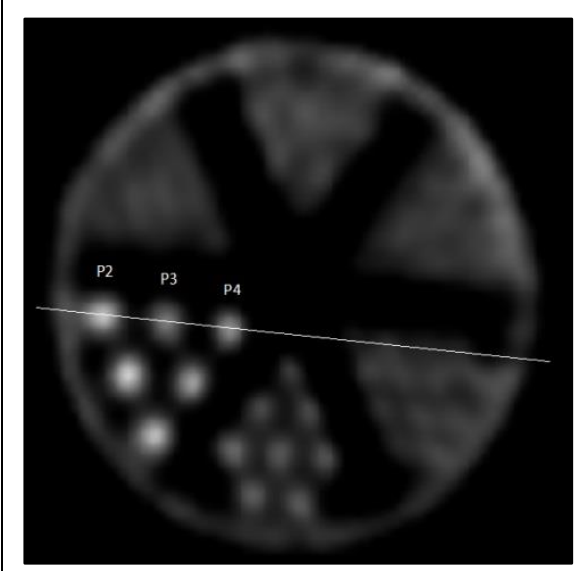

A

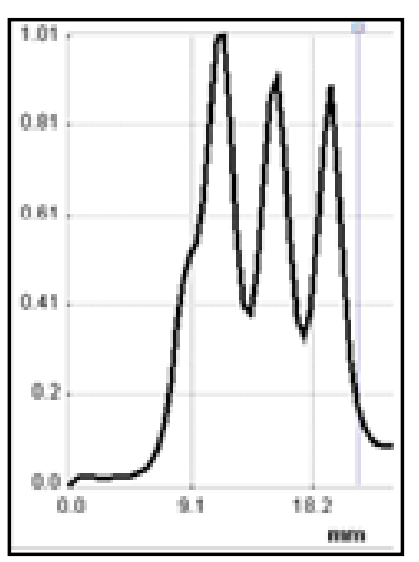

B

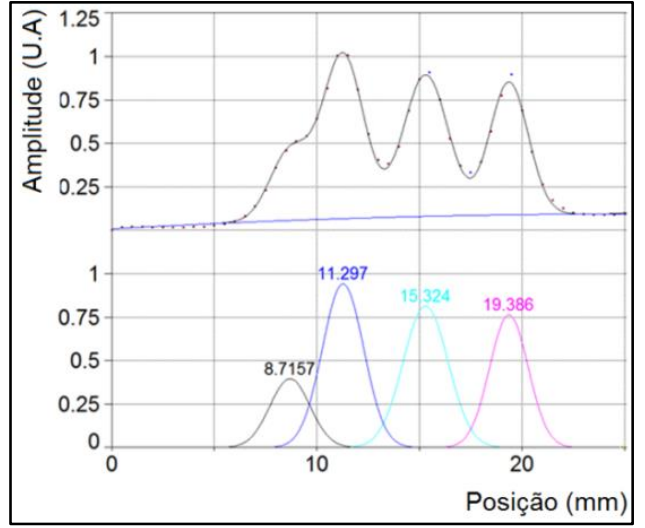

$\mathrm{C}$

Table 4 consolidates the results obtained after peaks analysis of line profiles from Groups G1, G2 and G3. Peaks referring to fillable channels from the others group (G4 to G6) cannot be resolved. This result is an indicative of the limitations in the spatial resolution of the PET scanner.

Table 4: Peaks analysis results of line profiles from Groups G1, G2 and G3.

\begin{tabular}{c|c|c|c|c}
\hline Group - Diameter (mm) & Peak & Amplitude (U.A) & Position (mm) & FWHM (mm) \\
\hline \multirow{4}{*}{ G1 - 2,0 } & P2 & $0,94 \pm 0,10$ & $11,29 \pm 0,02$ & 2,37 \\
\cline { 2 - 5 } & P3 & $0,81 \pm 0,01$ & $15,32 \pm 0,01$ & 2,58 \\
\cline { 2 - 5 } & P4 & $0,76 \pm 0,01$ & $19,38 \pm 0,01$ & 2,20 \\
\hline \multirow{3}{*}{ G2-1,5 } & P2 & $0,54 \pm 0,01$ & $13,90 \pm 0,04$ & 2,55 \\
\cline { 2 - 5 } & P3 & $0,44 \pm 0,01$ & $17,04 \pm 0,05$ & 2,33 \\
\cline { 2 - 5 } & P4 & $0,38 \pm 0,01$ & $19,92 \pm 0,04$ & 1,82 \\
\hline \multirow{5}{*}{ G3-1,2 } & P2 & $0,42 \pm 0,04$ & $9,39 \pm 0,33$ & 3,12 \\
\cline { 2 - 5 } & P3 & $0,31 \pm 0,22$ & $11,94 \pm 0,29$ & 2,33 \\
\cline { 2 - 5 } & P4 & $0,50 \pm 0,02$ & $14,71 \pm 0,21$ & 3,29 \\
\cline { 2 - 5 } & P5 & $0,22 \pm 0,14$ & $17,34 \pm 0,24$ & 1,99 \\
\hline
\end{tabular}


In the Hot Rod phantom, the spacing between the channels in each group corresponds to twice the diameter of the channel. The distances between channels in groups G1, G2 and G3 are 4.0, 3.0 and $2.4 \mathrm{~mm}$ respectively. An analysis of Table 3 reveals that the distance obtained for the G1 group was $(4.05 \pm 0.02) \mathrm{mm}$, for the G2 group it was $(3.01 \pm 0.18) \mathrm{mm}$ and for the G3 Group it was (2.65 $\pm 0.11) \mathrm{mm}$. These results correspond to the expected values and validate the analysis of the peaks.

The results for the test using the Hot- Rod phantom indicate that the spatial resolution of the equipment is around $1.2 \mathrm{~mm}$. This value corresponds to the diameter of the G3 group. For the G4 group (1.0 mm diameter), the equipment was not able to distinguish clearly the rod structures.

\subsection{Point Source}

Figure 7 presents PET images of ${ }^{22} \mathrm{Na}$ point source obtained after reconstructions by MLEM-3D and FBP-2D methods. In this Figure, the ${ }^{22} \mathrm{Na}$ point source images at each measurement positions were sequentially superimposed, from left to right according to the radial dislocation of the source. All images are presented at the same colour scale (omitted in Figure). A qualitative analysis of Figure 7 reveals that FBP images are more blurry when compared to MLEM images.

Figure 7: PET images (axial view) of the ${ }^{22} \mathrm{Na}$ point source at the axial centre of the FOV (top) and at one-fourth of the axial FOV from the centre of the axial FOV (bottom). Radial distances from the centre of the FOV are 0, 5, 10, 15 and $25 \mathrm{~mm}$ (left to right). All images are presented using the same colour scale.
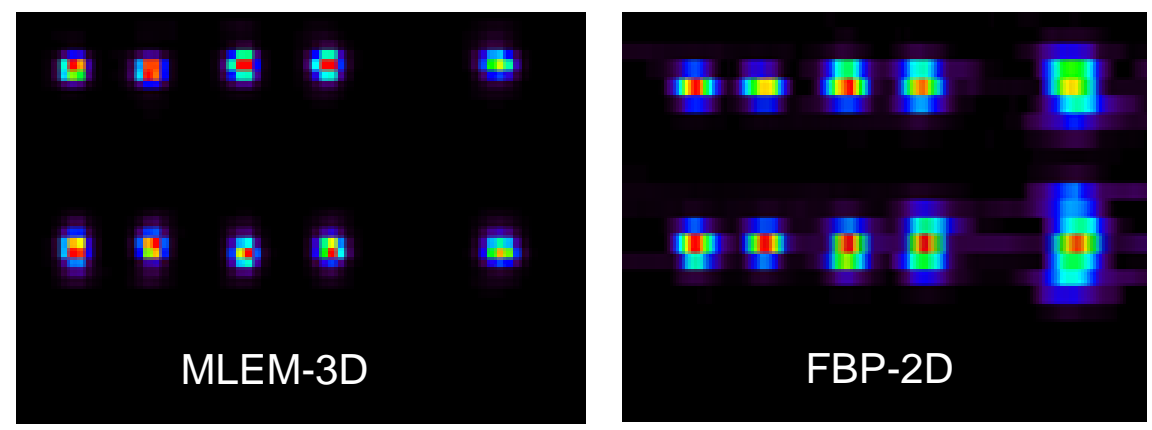

PET images of the ${ }^{22} \mathrm{Na}$ point source were analyzed in the radial, axial and tangential directions. Figure 8 illustrates the obtaining and analysis of the line profile of the ${ }^{22} \mathrm{Na}$ point source in the radial direction using Amide software. The same steps were adopted for the other two directions. 
Figure 8: Analysis of the ${ }^{22}$ Na point source PET image (Amide software).

A: Definition of the line passing through the centre of the image.

$B$ : Line profile of ${ }^{22}$ Na point source PET image.

$C$ : Gaussian fit of the line profile (obtaining FWHM e FWTM values).

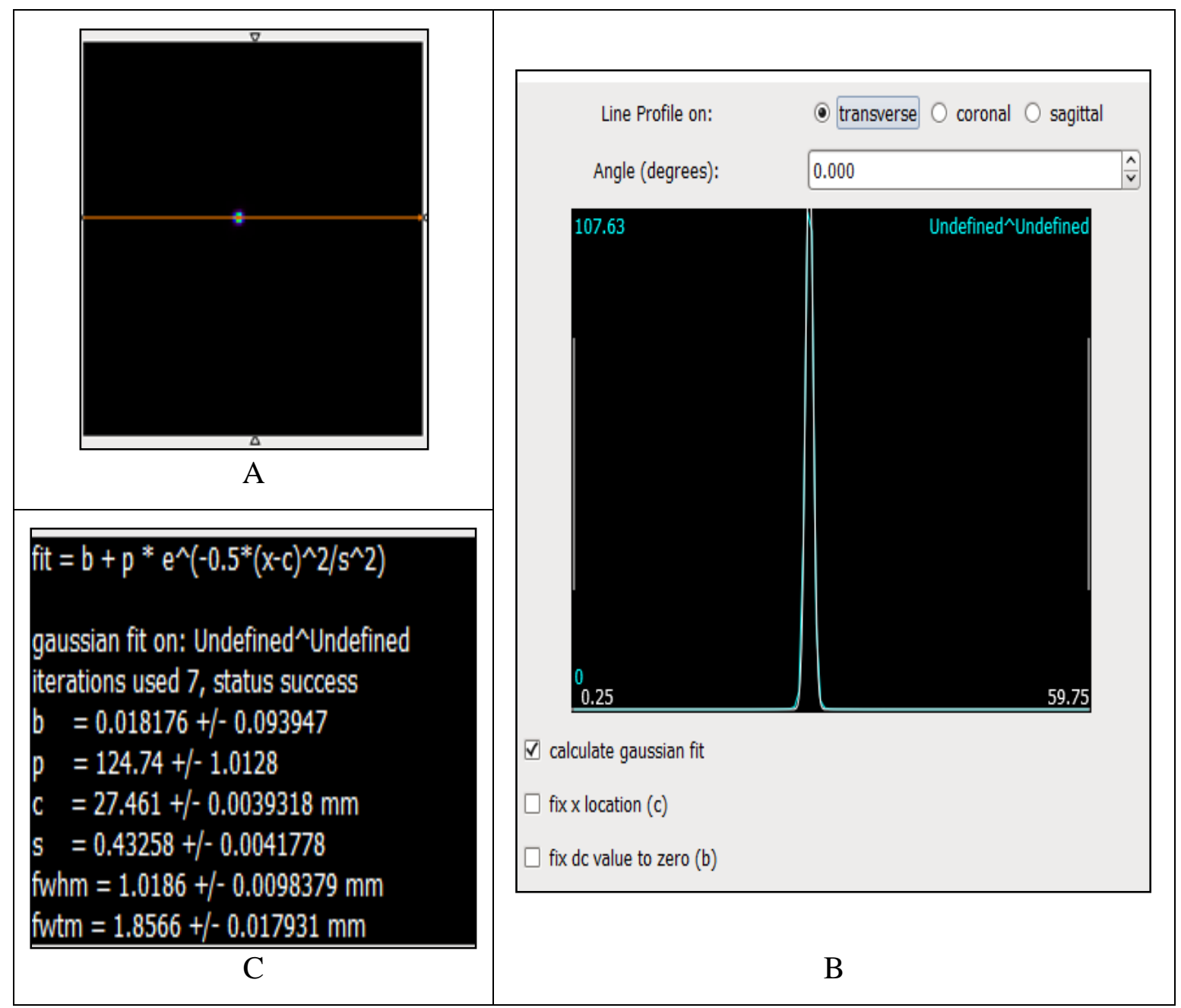

Figures 9 and 10 presents FWHM and FWTM results obtained after Amide analysis of the ${ }^{22} \mathrm{Na}$ point source PET images. In these figures, results for both reconstruction methods (FBP-2D and MLEM-3D) are showed - error bars were omitted. Roughly, for MLEM-3D images, FWHM and FWTM uncertainties were around 2\%, independently of the axial positioning or radial displacement of the source. For FBP-2D images, FWHM and FWTM uncertainties increased with the radial displacement of the source from around $5 \%(0 \mathrm{~mm})$ to $10-12 \%(25 \mathrm{~mm})$ in radial and tangential directions while in the axial direction these values ranged from $10 \%(0 \mathrm{~mm})$ to $20-24 \%$ (25 $\mathrm{mm}$ ). 
Figure 09: FWHMs of PET images of ${ }^{22} \mathrm{Na}$ source positioned at the axial centre of the FOV (CFOV) and at one-fourth of the axial FOV from the centre of the axial FOV (1/4 CFOV).

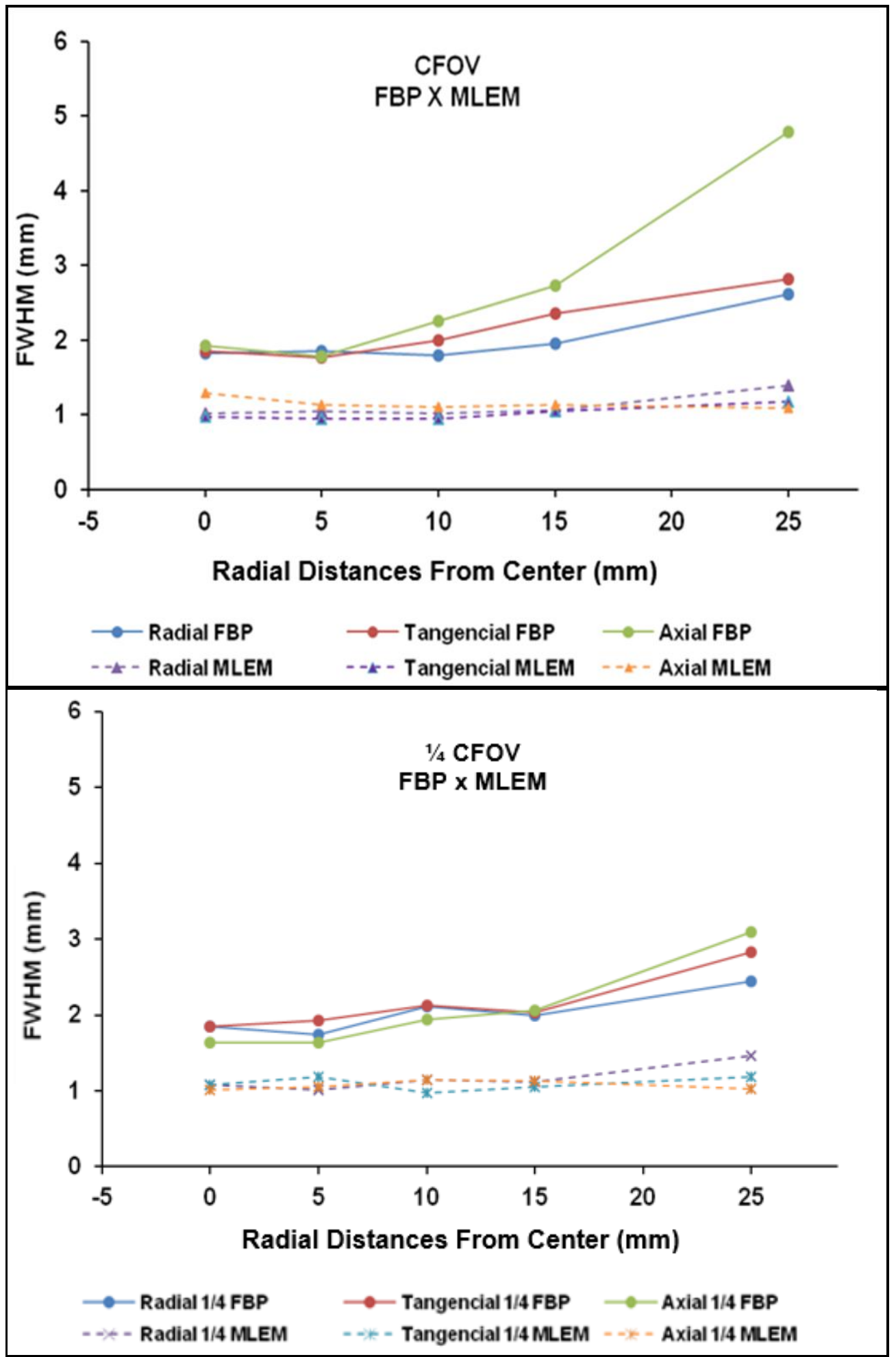


Figure 10: FWTMs of PET images of ${ }^{22}$ Na source positioned at the axial centre of the FOV (CFOV) and at one-fourth of the axial FOV from the centre of the axial FOV (1/4 CFOV).
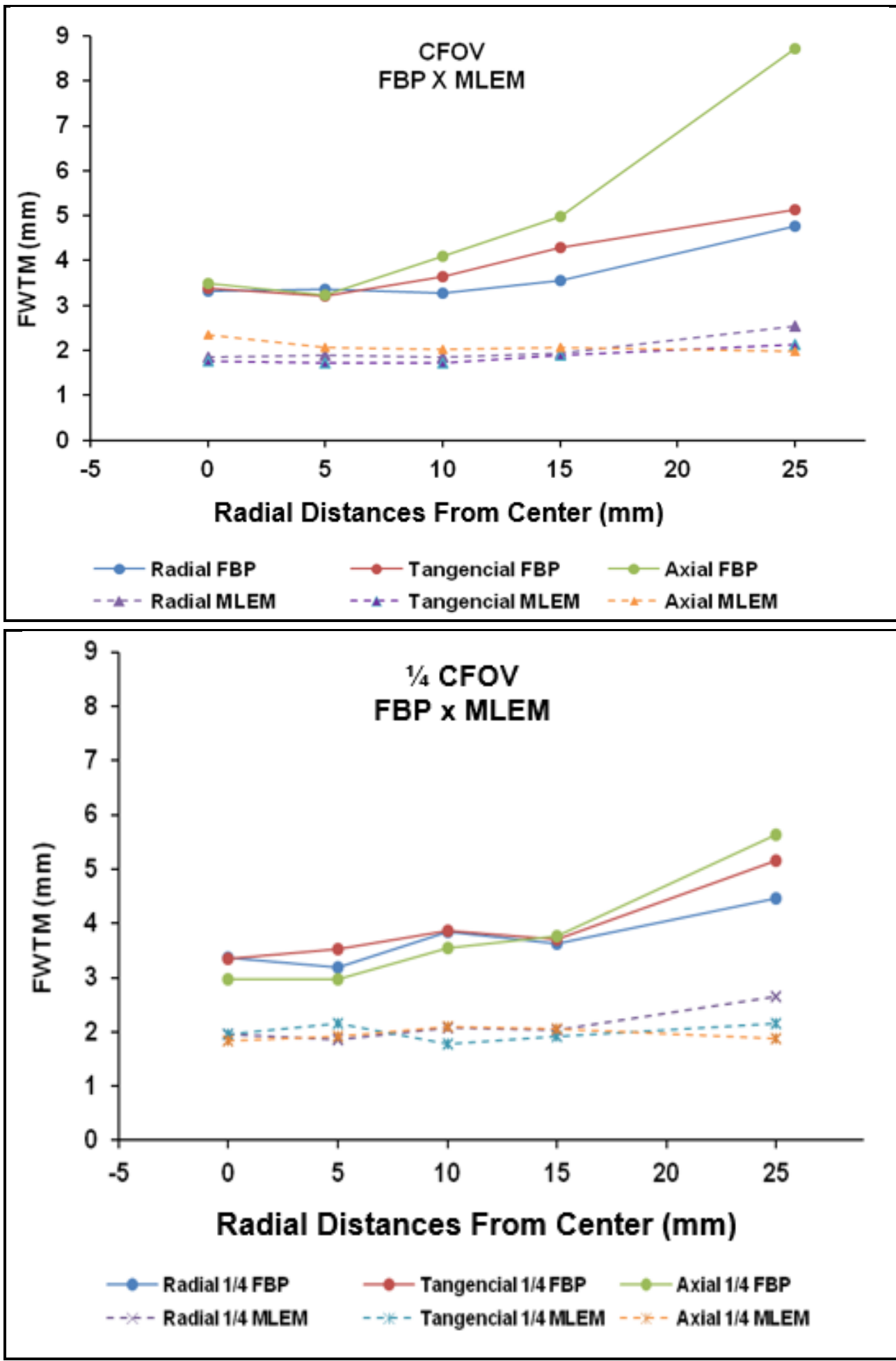
Figures 9 and 10 reveals that values for FWHM and FWTM are smaller when the PET images were reconstructed with the MLEM-3D method in comparison to the FBP-2D. This found was also observed in other works $[9,15]$ and was expected. As pointed early, the FBP algorithm introduces inaccuracies and artefacts that degrade the spatial resolution and make it uneven across the FOV when crystal spacing is irregular, as in LabPET SOLO 4. FBP spatial resolution degradation was particularly observed for axial resolution depending on the radial dislocation of the ${ }^{22} \mathrm{Na}$ source.

FBP results for spatial resolution considering different radial positions in the axial centre of the PET scanner are compatible (Table 5) with those from a precedent work dealing with the same PET scanner model [8]. The absence of published results for the 1/4-axial-offset position makes comparison impossible. Table 5 shows that FBP-2D spatial resolution values of LIM/CDTN PET scanner are slightly larger than those reported in the literature. The main differences $(>20 \%)$ were observed for the radial position $0 \mathrm{~mm}$. This fact requires further studies to be explained.

Table 5: Tangential and radial resolution with $F B P$ reconstruction of the ${ }^{22} N a$ point source PET image (source positioned at the axial centre of the FOV).

\begin{tabular}{|c|c|c|c|c|c|c|}
\hline \multicolumn{7}{|c|}{ Tangential Spatial Resolution } \\
\hline \multirow{2}{*}{$\begin{array}{c}\text { Radial } \\
\text { Position } \\
(\mathrm{mm}) *\end{array}$} & CDTN & Bergeron [8] & Diff(\%) & CDTN & Bergeron [8] & Diff(\%) \\
\cline { 2 - 7 } & $1.86 \pm 0.09$ & 1.28 & 45 & $3.38 \pm 0.16$ & 2.47 & 37 \\
\hline 0 & $1.76 \pm 0.08$ & 1.78 & -1 & $3.21 \pm 0.15$ & 2.97 & 8 \\
\hline 5 & $2.00 \pm 0.12$ & 1.68 & 19 & $3.65 \pm 0.21$ & 3.05 & 20 \\
\hline 10 & & Radial Spatial Resolution & & \\
\hline \multirow{2}{*}{$\begin{array}{c}\text { Radial } \\
\text { Position } \\
(\mathrm{mm}) *\end{array}$} & CDTN & Bergeron [8] & Diff(\%) & CDTN & Bergeron [8] & Diff(\%) \\
\cline { 2 - 8 } & $1.83 \pm 0.09$ & 1.42 & 29 & $3.33 \pm 0.17$ & 2.43 & 37 \\
\hline 0 & $1.85 \pm 0.08$ & 1.67 & 11 & $3.37 \pm 0.15$ & 3.10 & 9 \\
\hline 5 & $1.79 \pm 0.11$ & 1.81 & -1 & $3.27 \pm 0.19$ & 3.56 & -8 \\
\hline 10 &
\end{tabular}

* Table shows only results for coincident radial positions in both studies. 
In a general way, for MLEM-3D images, FWHM values were around 1mm while FWTM values were around 2mm (Figures 9 and 10). Differently from FBP-2D images, relevant differences were not observed for MLEM-3D images when the source was radially displaced at the axial centre of the FOV or at one-fourth of the axial FOV from the centre of the axial FOV. This behaviour was observed in all three directions.

The volumetric spatial resolution at the center of the FOV was $1.28 \mathrm{~mm}^{3}((1.09 \times 0.97 \times 1.29)$ $\mathrm{mm}^{3}$ ) for MLEM-3D reconstructions while for FBP-2D it was $6.64 \mathrm{~mm}^{3}((1.83 \times 1.86 \times 1.92)$ $\mathrm{mm}^{3}$ ). The volumetric resolution is defined as the product of radial, tangential and axial resolutions [14]. The MLEM-3D result is more compatible with those obtained with the Hot Rod phantom (that indicated a spatial resolution around $1.2 \mathrm{~mm}$ ) then the FBP-2D result.

It is important to note that evaluating spatial resolution using the ${ }^{22} \mathrm{Na}$ point source does not represent a routine condition of imaging usual objects such as small animals. It is just a special condition which makes comparisons between different PET scanner possible and reproducible [7]. Measurements with the Hot Rod phantom are closer to preclinical practices where different hot structures are presents in the subject and there is a significant tissue scatter.

Although NEMA NU 4-2008 publication presents standard methodologies to characterize small animal PET scanners performance, a recent paper analyzes its suitability [16]. The work presents a consistent discussion about the method of characterizing spatial resolution, points out potential flaws in the publication as well as suggests improvements.

\section{CONCLUSION}

This work carried out a systematic study of the spatial resolution of the LIM/CDTN small animal PET scanner. The absence of similar works in the literature made it impossible to perform a comprehensive comparison of the results with other authors.

Spatial resolution tests performed in this study were incorporated into the Quality Assurance Program (QAP) for Molecular Images of the LIM/CDTN with a semiannual frequency. 


\section{ACKNOWLEDGMENT}

The authors thank the CDTN/CNEN, FAPEMIG and CNPq for funding this work.

\section{REFERENCES}

[1] SAHA, G. B., Basics of PET Imaging: Physics, Chemistry, and Regulations, New York: Springer-Verlag, 2010.

[2] WU, H.; PAL, D.; SONG, T. Y.; O’SUlliVAN, J.; TAI, Y. Micro Insert: A Prototype FullRing PET Device for Improving the Image Resolution of a Small Animal PET Scanner, J Nucl Med, v. 10, p. 1668-1676, 2008.

[3] MOSES, W.W. Fundamental limits of spatial resolution in PET. Nucl Instrum Methods Phys Res A, 648 Supplement 1:S236-S240, 2011.

[4] GONG, K.; CHERRY, S. R.; QI, J. On the assessment of spatial resolution of PET systems with iterative image reconstruction. Phys Med Biol, v. 7, p. 61-65, 2016.

[5] GONZÁleZ, A. J.; AGUILAR, A.; CONDE, P.; HERNÁNDEZ, L.; MOLINER, L.; VIDAL, L. F.; SÁNCHEZ, F.; SÁNCHEZ, S.; CORRECHER, C.; MOLINOS, C.; BARBERÁ, J; LANKES., K; JUNGE., S; BRUCKBAUER, T.; BRUYNDONCKX, P.; BENLLOCH, J. M. A PET design based on SiPM and monolithic LYSO Crystals: Performance Evaluation. IEEE Trans Nucl Sci, v. 63, n. 5, p. 2471-2477, 2016.

[6] KRISHNAMOORTHY, S.; BLANKEMEYER, E; MOLLET, P; SURTI, S; VAN HOLEN, R; KARP, J. S. Performance evaluation of the MOLECUBES $\beta$-CUBE-a high spatial resolution and high sensitivity small animal PET scanner utilizing monolithic LYSO scintillation detectors. Phys Med Biol, v. 63, n. 15, 155013, 2018.

[7] NEMA, National Electrical Manufacturers Association. NEMA Standards Publication NU 42008 Performance measurements of small animal PET. Rosslyn: NEMA, 2008. 
[8] BEGERON, M.; CADORETTE, J.; BUREAU-OXTON, C.; BEAUDOIN, J. F.; TÉTRAULT, M. A.; LEROUX, J. D.; LEPAGE, M. D.; ROBERT, G.; FONTAINE, R.; LECOMTE, R. Performance Evaluation of the LabPET APD-Based Digital PET Scanner. IEEE Trans Nucl Sci, v. 56, n. 1, p. 10-16, 2009.

[9] PRASAD, H.; RATIB, O.; ZAIDI, H. NEMA NU-04-based performance characteristics of the LabPET- ${ }^{\text {TM }}$ small animal PET scanner, Phys Med Biol, v. 56, p. 6649-6664, 2011.

[10] GOERTZEN A. L.; BAO, Q.; BERGERON, M.; BLANKEMEYER, E.; BLINDER, S.; CANADAS, CHATZIIOANNOU, A. F.; DINELlE, K.; ELHAMI, E.; JANS, H. S.; LAGE, E.; LECOMTE, R.; SOSSI, V.; SURTI, S.; TAI, Y. C.; VAQUERO, J. J.; VICENTE, E.; WILLIAMS, D. A.; LAFOREST, R. NEMA NU 4-2008 comparison of preclinical PET imaging systems. J Nucl Med, v.53, n.8, p.1300-1309, 2012.

[11] BEGERON, M.; CAdORETTE, J.; TÉTRAult, M. A.; BEAUdOIN, J. F.; LEROUX, J. D.; FONTAINE, R.; LECOMTE, R. Imaging performance of LabPET APD-based digital PET Scanners for pre-clinical research. Physics in Medicine and Biology, v.59, p.661-678, 2014

[12] GONTIJO, R. M .G.; FERREIRA A. V.; SILVA, J. B.; MAMEDE M. Quality control of small animal PET scanner: the Brazilian scenario. Braz J Rad Sci, v. 8, n. 2, p 1-9, 2020.

[13] GE HEALTHCARE, Triumph Service Guide, Technical Publication, Revision Draft 6, USA: GE Healthcare, 2011. 524p.

[14] QMR - Quality Assurance in Radiology and Medicine. Micro-PET Hot-Rod Phantom, Available at: http://www.qrm.de/content/pdf/QRM-MicroPET-Hot Rod.pdf . Last accessed: 24 Jan. 2020.

[15] YANG,Y.; TAI, Y. C.; SIEGEL, S.; NEWPORT,D. F.; BAI, B.; LI, Q.; LEAHY, R. M.; CHERRY, S. R. Optimization and performance evaluation of the microPET II scanner for in vivo small-animal imaging. Phys Med Biol, v. 49, p. 2527-2545, 2004.

[16] HALLEN, P.; SCHUG, D.; SCHULZ, V. Comments on the NEMA NU 4-2008 standard on performance measurement of small animal positron emission tomographs. EJNMMI Phys, v.7, n.12, p. 1-20, 2020. 